\title{
New insights into the functioning and structure of the PE and PP plastispheres from the Mediterranean Sea ${ }^{\text {tr }}$
}

\author{
A. Delacuvellerie ${ }^{\text {a }}$, A. Géron ${ }^{\mathrm{a}, \mathrm{b}}, \mathrm{S}$. Gobert ${ }^{\mathrm{c}, \mathrm{d}}$, R. Wattiez ${ }^{\mathrm{a}, *}$ \\ ${ }^{a}$ Proteomics and Microbiology Department, University of Mons, 20 Place du Parc, 7000, Mons, Belgium \\ ${ }^{\mathrm{b}}$ University of Stirling, Division of Biological and Environmental Sciences, Faculty of Natural Sciences, FK9 4LA, Stirling, UK \\ c Oceanology Department, University of Liège, 11 Allée du 6 Août, 4000, Liège, Belgium \\ d STAtion de REcherches Sous-marines et Océanographiques (STARESO), Punta Revellata, BP33, 20260, Calvi, France
}

\section{A R T I C L E I N F O}

\section{Keywords:}

Plastic debris

Functional role

Biofilm

Metaproteomic analysis

Metagenome

Marine environment

\begin{abstract}
A B S T R A C T
Plastic debris are accumulating in the marine environment and aggregate microorganisms that form a new ecosystem called the plastisphere. Better understanding the plastisphere is crucial as it has self-sufficient organization and carries pathogens or organisms that may be involved in the pollutant adsorption and/or plastic degradation. To date, the plastisphere is mainly described at the taxonomic level and the functioning of its microbial communities still remains poorly documented. In this work, metagenomic and metaproteomic analyzes were performed on the plastisphere of polypropylene and polyethylene plastic debris sampled on a pebble beach from the Mediterranean Sea. Our results confirmed that the plastisphere was organized as self-sufficient ecosystems containing highly active primary producers, heterotrophs and predators such as nematode. Interestingly, the chemical composition of the polymer did not impact the structure of the microbial communities but rather influenced the functions expressed. Despite the fact that the presence of hydrocarbon-degrading bacteria was observed in the metagenomes, polymer degradation metabolisms were not detected at the protein level. Finally, hydrocarbon degrader (i.e., Alcanivorax) and pathogenic bacteria (i.e., Vibrionaceae) were observed in the plastispheres but were not very active as no proteins involved in polymer degradation or pathogeny were detected. This work brings new insights into the functioning of the microbial plastisphere developed on plastic marine debris.
\end{abstract}

\section{Introduction}

Plastic production increases every year and generates large amount of plastic waste, e.g. $302 \mathrm{Mt}$ in 2015, of which only a small fraction is recycled (Geyer et al., 2017). Plastic waste accumulates in landfills and in most environmental compartments (e.g., marine environment, freshwater, in the soil) (Geyer et al., 2017). The Mediterranean sea has one of the world's highest sea plastic densities (Galgani et al., 2000; Munari et al., 2017), containing between 1000 and 3000 tons of floating plastics in 2013 (Cózar et al., 2015). Microorganisms rapidly colonize the surface of these synthetic polymers once they enter in the marine environment. This process is facilitated by species producing an extracellular matrix forming a biofilm. This distinct environmental niche is called the "plastisphere" (Zettler et al., 2013). Better understanding the plastisphere is of immediate importance for several reasons. Firstly, plastics act as free substrates allowing microorganism colonization and self-sufficient ecosystem formation, which can contain invasive species or cause bloom of harmful algae (Barnes, 2002; Garcés \& Camp, 2003). Secondly, floating plastics can be used as a vessel by pathogenic bacteria, such as Vibrio, to navigate across the oceans, increasing their dispersion power (Debroas et al., 2017; Oberbeckmann et al., 2016; Zettler et al., 2013). Finally, microorganisms present in the plastisphere can be involved in synthetic polymer degradation or organic pollutant absorption (Oberbeckmann et al., 2016; Zettler et al., 2013).

The bacterial communities of the plastisphere have been found to be distinct and metabolically more active from surrounding sediment or water (De Tender et al., 2015; Zettler et al., 2013; Bryant et al., 2016). Some studies suggested that the bacterial composition depends on the polymer type, while others reported no difference. In fact, the communities growing on different polymers are distinct in the early biofilm

\footnotetext{
This paper has been recommended for acceptance by Maria Cristina Fossi.

* Corresponding author.

E-mail address: ruddy.wattiez@umons.ac.be (R. Wattiez).
} 
stage formation (after two weeks), while they become similar in the later stages, with only few specific bacteria depending on the plastic chemical composition (Kirstein et al.,2018; Oberbeckmann et al., 2016; Pinto et al., 2019). Two recent metagenomic studies compared the composition of the microbial communities on non-biodegradable plastic versus a control surface (i.e. ceramic) and non-biodegradable polymer versus biodegradable plastic (Bryant et al., 2016) (Pinnell et al., 2019). While the communities from the non-biodegradable plastic showed no difference with the control (Bryant et al., 2016), the second study demonstrated that biodegradable plastics select microorganisms involved in polymer degradation and in sulfate reduction (Pinnell et al., 2019). Several studies have reported the presence of pathogenic bacteria on plastics including Vibrio parahaemolyticus (Kirstein et al., 2016); Tenacibaculum genus (Oberbeckmann et al., 2016) and finally, the pathogen Aeromonas salmonicia (Virsek et al., 2017). This confirmed that plastics serve as a vector for the transport of microorganisms in environments where they are not usually detected (De Tender et al., 2015), which can lead to bird and fish disease propagation (Zettler et al., 2013). Key species seem to be selected on the plastics for their capacity to degrade the plastic or pollutants absorbed on the plastic surface, as shown by the presence of Alcanivorax or Hyphomonas, Oceaniserpentilla genus, hydrocarbon-degrading bacteria (Oberbeckmann et al., 2016; Zettler et al., 2013).

Recent studies provided first insights into the metaproteome of the plastisphere (Oberbeckmann et al., 2021). They highlighted that the microorganisms were rather more influenced by the geological parameters than plastic chemical composition (polyethylene (PE) or polystyrene (PS)) and were not implicated in their degradation. Moreover, in the photic zone, phototrophic organisms such as diatoms and Cyanobacteria were found to be opportunistic colonizers of the plastic surface. In contrast with studies based on the $16 \mathrm{~S}$ rRNA sequencing, plastics were not carriers of harmful microorganisms such as Vibrio pathogens.

In this original work, polypropylene (PP) and PE macroplastic biofilms sampled on a pebble beach in the Mediterranean Sea (Calvi, Corsica) were analyzed using metagenomic and metaproteomic. This study deciphered for the first time the bacterial community structure and functioning of the plastisphere depending on the plastic chemical composition.

\section{Materials and methods}

\subsection{Sampling}

Macroplastics were collected in May 2019 (Table S1) on the coastline of the Mediterranean Sea (Corsica, Calvi) along Oscellucia beach (Figs. S1 and $42.573,689^{\circ} \mathrm{N}, 8.724537^{\circ} \mathrm{E}$ ). Plastics were sampled manually on the pebbles that were continuously swept by the sea water. Water salinity, $\mathrm{pH}$ and temperature were $38,8.4$ and $17.8{ }^{\circ} \mathrm{C}$, respectively. The plastic samples were conserved in sterile tube containing sea water, stored at $4{ }^{\circ} \mathrm{C}$ during the transport. Prior to DNA and protein extraction, the plastic samples were rinsed with sterile marine water (38 $\mathrm{g} / \mathrm{L}$, Sigma Sea Salt) in order to remove the free-living microorganism fraction.

\subsection{ATR-FTIR}

The chemical composition of plastic samples was identified by Fourier transform infrared spectroscopy (FTIR) using the attenuated total reflectance (ATR) technique (Bruker, Tensor 27) with OPUS 6.5 software (Fig. S2). The polymer surface was cleaned with ethanol $70 \%$ $(\mathrm{V} / \mathrm{V})$ and dried. The spectrum of the film was acquired over the wavelength range of $4000-600 \mathrm{~cm}^{-1}$ with 64 spectral scans. Five pieces of plastics were in PP and four in PE.

\subsection{Plastic sample processing}

Five PP and four PE samples with a well-developed biofilm were selected for downstream metagenomic and the metaproteomic analyses. Plastic biofilms were scrapped with a sterile scalpel blade to recover a maximum of biomass. This biomass was separated in two: the first part was used for the DNA extraction and the second for the protein extraction. The biomass was centrifugate at $16,000 \mathrm{~g}$ at $4{ }^{\circ} \mathrm{C}$ to obtain pellets.

\subsection{DNA extraction}

The plastic biofilms were scrapped with a sterile scalpel blade to ensure maximum biomass recovering. DNA was extracted using the biofilm DNA isolation kit (NORGEN BIOTEK CORP. ()) following the manufacturer's instructions. DNA was sequenced by EUROFIN GENOMICS $\odot$ using the shotgun analysis by Illumina paired end $(2 \times 150 \mathrm{pb})$. Metagenomic sequences were deposited at the SRA (Sequence Read Archive) in NCBI under the accession number PRJNA770505 (http:// www.ncbi.nlm.nih.gov/bioproject/770505).

\subsection{Metagenomic analysis}

The sequence quality was assessed by the removal of poor-quality bases. Bases were removed from the $3^{\prime}$ and $5^{\prime}$ if the average phred quality was below 15 using a sliding window approach. Only mate pairs (forward and reverse) were used for the downstream analysis. This step was performed by EUROFIN GENOMICS. OTUs were selected at a sequence identity level of $97 \%$ using the refseq data-base on MGRast (Pruitt et al., 2007; Glass et al., 2010). The quality of the metagenomic sequencing was assessed by performing rarefaction curves, and these were performed using the PAST software (Hammer et al., 2001) (Fig. S3). The data were rarefied at 26,255 reads (Table S2) using the Limma RGUI package (Ritchie et al., 2015). The alpha-diversity indices (Shannon index and OTU richness) were calculated using PAST software (Hammer et al., 2001). The vegan RGUI package (Wang et al., 2012) was used to realize multivariate analysis using the PERMONOVA test on the Bray-Curtis dissimilarity using 10,000 permutations (Table 1). The presence of human pathogenic bacteria was investigated using the Bode Science Center database (https://www.bode-science-center.com/cente r/relevant-pathogens-from-a-z.html).

\subsection{Protein extraction and mass spectrometry analysis}

Protein extraction was performed on the biomass pellets using the protocol as described in Leroy et al. (2015). After trypsin digestion, the samples were concentrated and evaporated with the Speed Vac and the pellets were resuspended in $50 \mu \mathrm{l}$ of loading buffer ( $2 \%$ acetonitrile (ACN) and $0.1 \%$ formic acid). Peptides were quantified using the PierceTM Quantitative Colorimetric Peptide Assay (Thermo Fisher Scientific (C). Peptides $(2.5 \mu \mathrm{g})$ were analyzed using a label-free strategy on an UHPLC-HRMS platform with an Eksigent 2D liquid chromatograph and an AB SCIEX Triple TOF 5600 using the protocol as described previously by Géron et al. (2019).

Table 1

PERMANOVA analysis using the Bray-Curtis dissimilarity with 10,000 permutations. One-way PERMANOVA analysis was performed using the Bray-Curtis dissimilarity with 10,000 permutations on the following factors: polypropylene (PP) $v s$ polyethylene (PE). Significance: *, $\mathrm{P}<0.05$; **, $\mathrm{P}<0.01$; ***, $\mathrm{P}<0.001$.

\begin{tabular}{lccc}
\hline Factors tested & $\mathrm{p}$ & $\mathrm{r}^{2}$ & Signif. \\
\hline One-way PERMANOVA & (Bray-Curtis & dissimilarity, & 10,000 permutations): \\
Conditions (PP, PE) & 0.5053 & 0.072 & $/$ \\
\hline
\end{tabular}




\subsection{Creation of database for metaproteomic analysis (mPies) and protein identification}

Protein search databases (DBs) were generated with the mPies v0.9 program using the non-assembled metagenome as a template for each sample (Werner et al., 2019). Briefly, mPies trimmed sequencing raw reads with Trimmomatic (Bolger et al., 2014) and predicted genes using FraGeneScan (Rho et al., 2010). Redundant proteins with a similarity of less than $80 \%$ were removed. Protein identification was performed using ProteinPilot (ProteinPilot software 4.5; Revision: 1656; Paragon Algorithm: 4.5.0.0, 1654; AB SCIEX, Framingham, MA, United States) (Matrix Science, London, United Kingdom; V. 2.2). Parameters used for the identification search were described as follows: Sample Type: Identification, Cys alkylation: Iodoacetamide, Digestion: Trypsin, ID Focus: Amino acid substitutions and Biological Modifications, Search effort: Thorough ID, Detected Protein Threshold (Unused ProtScore (Conf)) >: $0.05(10.0 \%)$. First identification searches were performed against the full size DBs. Then, second identification searches were performed against subset-DBs containing only the protein sequences that matched in the first-round searches. The FDR analysis was performed on the second-round search output with a global threshold of $1 \%$ calculated at the protein level. A validation by manual validation of the protein matches with only one peptide was performed to ensure that a series of at least five consecutive sequence-specific b- and y-type ions was observed.

\subsection{Protein annotation}

Identified proteins were annotated using the mPies program (Werner et al., 2019; Géron et al., 2019). Briefly, each identified protein sequence was aligned against the non-redundant UniProt DB (Swiss-Prot) and NCBI DB using Diamons (Buchfink et al., 2015). Up to 20 bests hits were retrieved based on the alignment score. For taxonomic annotation, the last common ancestor (LCA) among the bests hits was returned using MEGAN (bit score $>80$ ) (Huson et al., 2016). For functional annotation, the most frequent protein name was retained with a consensus tolerance threshold of similarity higher than $80 \%$ among the best blast hits. Proteins with a functional annotation score lower than $80 \%$ were manually validated (Table S4, Géron et al., 2019).

\subsection{Metaproteomic analysis}

Metaproteomic data were normalized using the total area sums. Regarding the statistical analysis of the proteomic results, Student's ttest and PCA were realized on MarkerViewTM 1.2.1 (ABSciex, United States). The relative abundance of some proteins significantly up or down regulated between our plastic types (PP, PE) was highlighted using the fold change. This score was determined through $t$-test with Pvalue $<0.05$. Only the proteins with a fold change $>1.5$ or $<0.6$ were considered as up or down-regulated, respectively.

Throughout our study, we used relative semi-quantitative analysis of the identified proteins (taxonomic or functional). Indeed, for each sample, the relative number of identified peptides was identified. This method does not take into account that some proteins are ionized more easily and may produce more peptides. The presence of human pathogenic bacteria was investigated using the Bode Science Center database (https://www.bode-science-center.com/center/relevant-pathoge ns-from-a-z.html).

\section{Results and discussion}

\subsection{Structure of the plastisphere revealed by metagenomic and metaproteomic analysis}

As revealed by the non-metric multidimensional scaling (nMDS) (Fig. S4) and PERMANOVA (Table 1) analyses, the polymer chemical composition was not found to significantly impact the metagenomic structure of the bacterial communities (Fig. 1C, Table S3). The alphadiversity analyses showed that the richness and the equitability indexes were smaller for the PP samples than PE despite the difference not being significative (Fig. S5). Even though the communities were not distinct, 85 and 155 OTUs were found to be specific to the PP and PE polymers, respectively.

On both PP and PE plastispheres, metagenomic reads were mainly characterized as Bacteria (91\%-99\%; Fig. 1a). Gammaproteobacteria was the most abundant class and was particularly represented $(>90 \%)$ on ZI_1, ZI_2 and ZI_3 samples. Alphaproteobacteria was found to be the second most represented class (50\% in the ZI_9 sample), followed by Cyanobacteria that reached $10 \%$ of the read abundance in the ZI_5 community. The most represented family was Vibrionaceae followed by Flavobacteriaceae (reached up to $89 \%$ in the ZI_3 sample and 62\% of total reads on ZI_8 samples, respectively) (Fig. S6B). The high representation of Alphaproteobacteria and Gammaproteobacteria in the plastisphere was consistent with previous studies (Delacuvellerie et al., 2019; Oberbeckmann et al., 2018).

Interestingly, the metaproteomic taxonomic profiles were found to be distinct from what resulted from the metagenomic analyses (Fig. 1C). The majority of the identified proteins (71-91\%) belong to the Bacteria domain for most samples (Fig. 1B). The ZI-1, ZI-6 and ZI-17 samples contained abundant Eukaryotic proteins (50-64\%). Cyanobacteria was the most represented phylum, followed by Alphaproteobacteria and Gammaproteobacteria. At the family level, Vibrionaceae was significantly less characterized than in the metagenomes, while the abundance of Rhodobacteraceae was consistent in both the read and protein number (Fig. S6B). Contrast in reads and protein abundance could suggest that Vibrionaceae were in a dormancy state in the plastisphere. Indeed, pebbles that are continuously swept by the waves consist in a constantly changing environment where a dormancy state can help pathogens like Vibrionaceae to deal with salinity, nutrient concentration, temperature and light gradients. In response to these changes, bacteria can shift their metabolism and enter a dormancy state, to survive and persist in the environment (Colwell and Huq, 1994).

In contrast with Vibrionaceae, Rhodobacteraceae was abundant in both reads and proteins (Fig. S6B). Rhodobacteraceae is known to be usually abundant in the plastisphere (Bryant et al., 2016; Ogonowski et al., 2018) and to play key role in the early stage of the biofilm formation. Rhodobacteraceae can colonize free plastic surface producing EPS (exopolysaccharide), which can facilitate the attachment of other organisms (Amaral-Zettler et al., 2020). Flavobacteriaceae, which is also part of the bacterial core of the plastisphere (De Tender et al., 2017; De Tender et al., 2015; Oberbeckmann et al., 2014), was found on 8 samples and reached $5 \%$ of the community's total protein. Flavobacteraceae are often found to be associated with diatoms and have previously been described as a key taxa into the biofilm for its ability to feed off exudates (Amin et al., 2012).

At the Eukaryote level (Fig. 1D), the taxonomic profiles derived from metaproteomic and metagenomic analysis were similar. Reads and proteins associated with unclassified organisms were dominant. The most abundant phylum was Bacillariophyta, a taxa that contains diatoms, followed by the Arthropoda in the metaproteomes and Phaeophyceae (brown algae) in the metagenomes. A recent metaproteomic study from Oberbeckmann and colleagues of the plastisphere from PS and PE immersed for 2 weeks on the coast showed similar results (Oberbeckmann et al., 2021). However, they observed higher Alphaproteobacteria abundance which can be explained by the fact that the immersion time of our sample is unknown and the bacterial community evolve gradually overtime (De Tender et al., 2017). Moreover, our plastics were in contact with pebbles, contrary to their study.

\subsection{Self-sufficient ecosystem}

As shown in the previous section (Fig. 1), the primary producers (i.e., 
A)

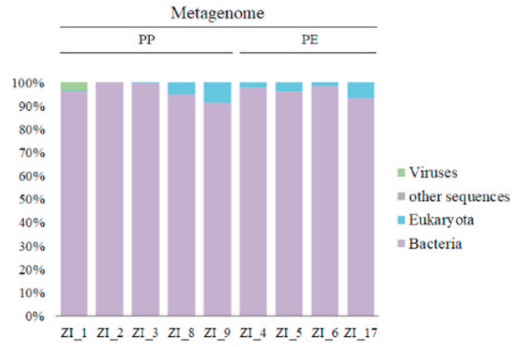

B)

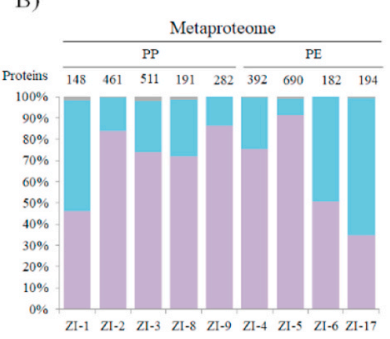

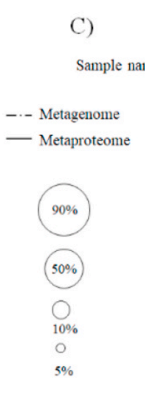

C)

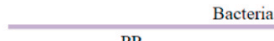

Bacteria
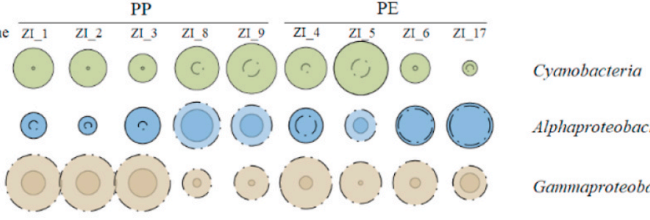

Alphaproteobacteria

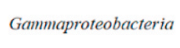

$\circ \circ \circ \circ \circ \circ \circ \circ \quad \circ \quad \circ \quad$ unclassified

$\circ$

- () $\odot$

0

Verrucomicrobia

Bacteroidetes

Betaproteobacteria



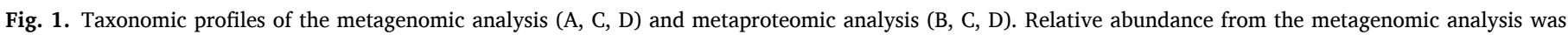

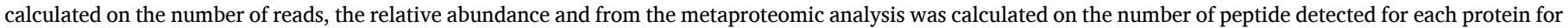
each sample.

Cyanobacteria and Bacillariophyta) were the most active organisms found in both PP and PE samples. The more common heterotrophic bacteria found on the plastic surface, Roseobacter, is also present on our samples. Moreover, in the plastisphere, the presence of some predators is known. Previous metagenomic analysis also highlighted the presence of some predators, such as Micromonas which are small flagellates (Amaral-Zettler et al., 2020). Here, no protein from Micromonas was found. These organisms are predators of bacteria and other microorganisms. Flavobacteraceae family present in our metaproteome (Fig. S6B) can contain some bacterial members able to lyse diatoms (Amin et al., 2012). All these results confirm previous studies claiming that plastic is a self-sufficient ecosystem containing phototrophs, heterotrophs and predators (Zettler et al., 2013; Amaral-Zettler et al., 2020). Most of this research is focused on the microorganisms. On the ZI-1 sample, we identified a protein with 5 peptides: a major sperm protein from an organism belonging to Rhabditidae (Nematoda). In addition to being a self-sufficient ecosystem with the presence of primary producers that are the most active organisms into the microbial community, the plastic surfaces seem to be a place for the reproduction of "bigger" organisms, such as nematodes.

The functions in the metagenomes and the metaproteomes showed contrasting distribution (Fig. S7). For example, while the functions involved in photosynthesis and in protein metabolism were highly represented in the metaproteomes, they were slightly characterized in the metagenomes. Metaproteomic analyses study the final genes products - proteins - and reveal the major microbial players of the microbial community, while metagenomics provide information on potentially expressed functions. Therefore, we decided to focus on the plastisphere metaproteomes to better understand the functional role of these microbial communities. At the functional level of phototroph microorganisms, the most abundant COG/KOG corresponded to the energy production and conversion (Fig. S8), including the proteins involved in photosynthesis. The relative abundance of the photosynthesis involved proteins was not found to depend on the plastic's chemical composition (Fig. 2). Phycobilisome involved proteins (including allophycocyanin and phycocyanin) were the most abundant proteins in Bacteria, while the PSI and PSII-related proteins were the most expressed in Eukaryotes. The proteins involved in photosynthesis were more abundant on the plastic surface than the surrounding environment (Bryant et al., 2016; Oberbeckmann et al., 2021). Cyanobacteria have an important role in the plastic biofilm by the contribution and implication in the net primary production positive (Bryant et al., 2016). Moreover, the high abundance of phycobilisome protein found in the plastisphere can be explained by the fact that Cyanobacteria seems to preferentially use phycobilisome complexes to absorb photons for photosynthesis than other Cyanobacteria species in the surrounding water column that use chlorophyll-binding complexes (Bryant et al., 2016). In combination with Cyanobacteria, Diatoms (Bacillariophyta) were phototrophs commonly found on the plastic surface exposed to the sunlight. These microorganisms are constitutive to plastic biofilms. The high proportion of diatoms seems to indicate a recent colonization of the plastic (Amaral-Zettler et al., 2020). Plastic debris represent a new inhabit and an inert surface in the photic zone of the marine environment, and organisms able to benefit from light, or which are protected from UV, colonize these new habitats.

\subsection{The influence of the plastic chemical composition}

As previously shown, the structure of microbial communities was not impacted by the polymer chemical composition (i.e,. PP vs PE; Fig. 1C, Table S3). Interestingly, previous studies showed contrasting results (Zettler et al., 2013; Debroas et al., 2017), while others were concomitant with our observations (Delacuvellerie et al., 2021; Oberbeckmann et al., 2021). Most protein functions were either associated to (i) 


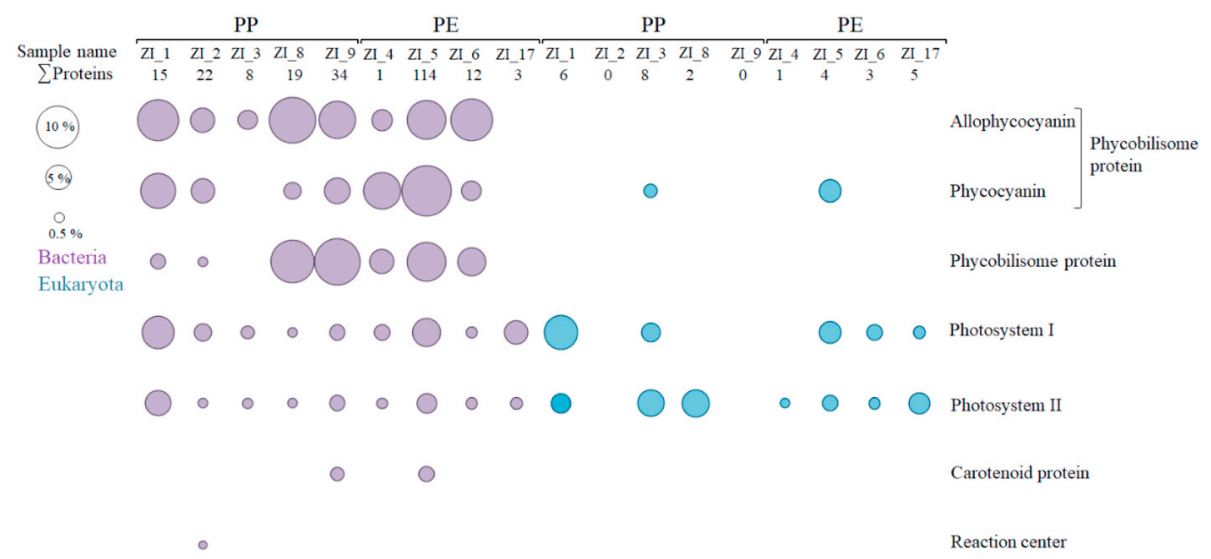

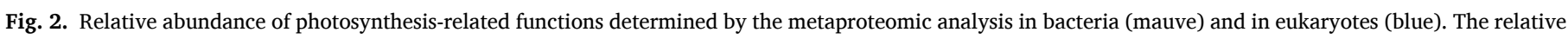

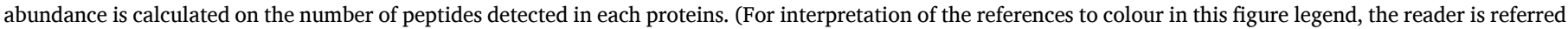
to the Web version of this article.)

translation, ribosomal structure and biogenesis, (ii) energy production and conversion, or (iii) carbohydrate transport and metabolism (Fig. 3B). Only 23 and 43 proteins were found to be up regulated on PP and PE, respectively (Fig. 3). These proteins were involved in the general metabolism, such as photosynthesis or cell transport. Proteins involved in energy production and conversion were upregulated on PE and most of these proteins belonged to Cyanobacteria (Fig. 3A), while proteins up regulated on PP mainly took part in translation, ribosomal structure and biogenesis metabolism and were mainly represented by Alphaproteobacteria and Cyanobaceria. In the recent metaproteomic analysis of Oberbeckmann and colleagues, only minor differences of bacterial composition were observed between PE and PS such as the presence of some Cyanobacteria families having more affinity with one plastic (Oberbeckmann et al., 2021). The characteristics of the surface polymer (i.e., the roughness, the hydrophobic surface or the crystallinity) may be responsible for the small differences of microbial composition between

A)

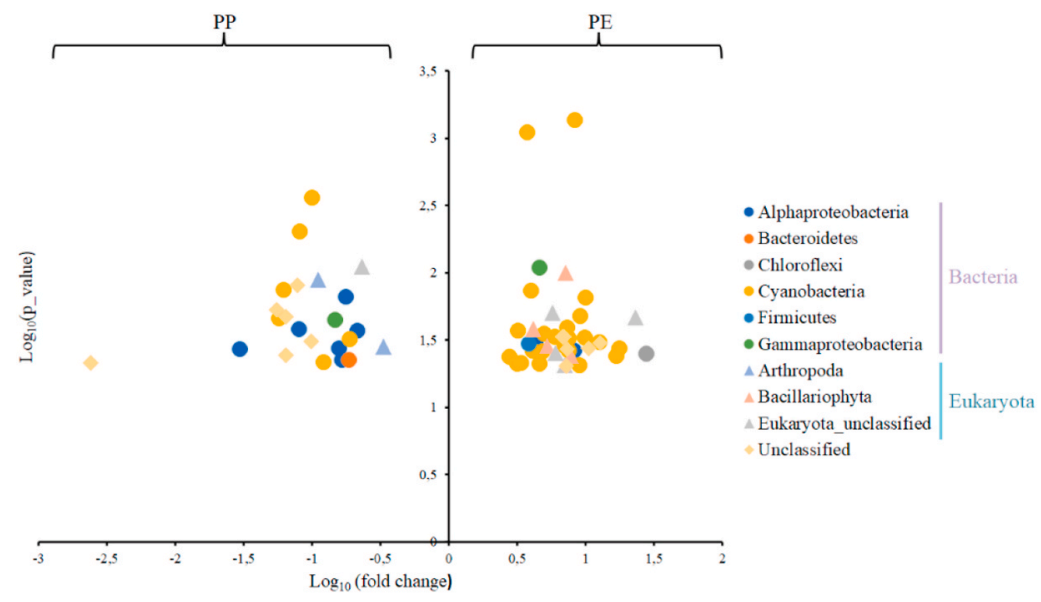

B)

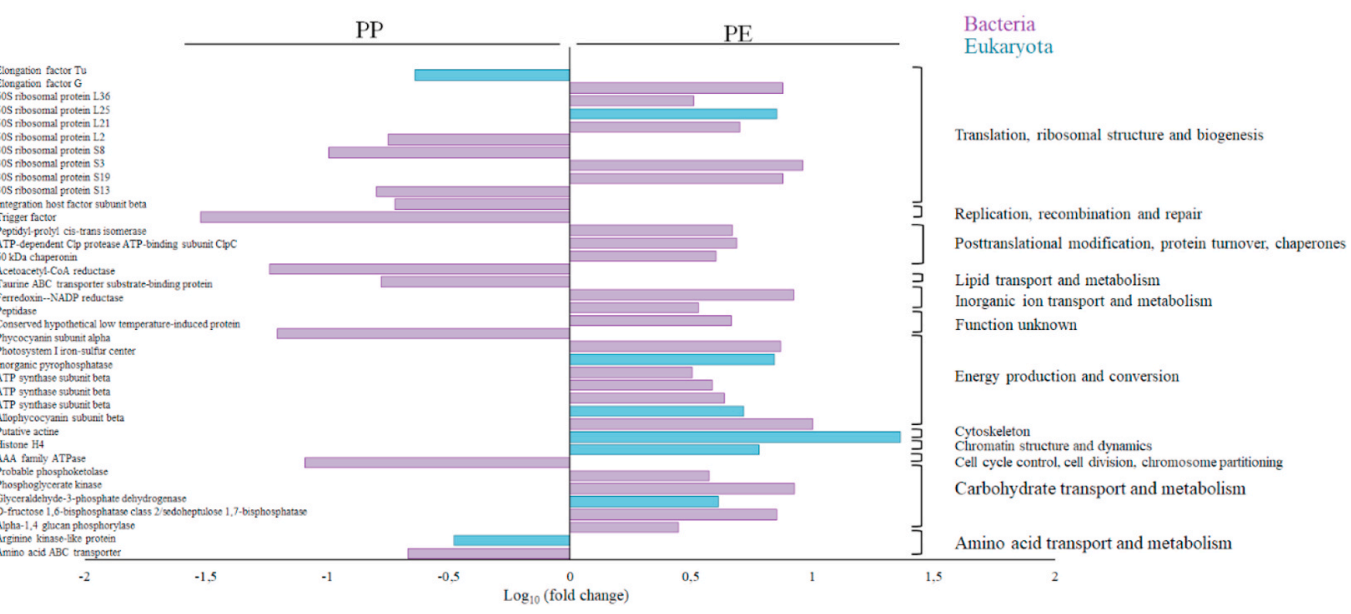

Fig. 3. Fold change of proteins significantly expressed on PP or on PE, (A) according to the taxonomic composition and (B) the protein function. 
PP and PE. However, PP and PE plastics have similar chemical structures, explaining the similarity between the microbial communities on PP and PE. Previous observations have highlighted that a "core" of bacteria was shared among all the polymers as a more general biofilm (Kirstein et al., 2018). Moreover, the plastic localization in the water column (sediment $v s$ water column) or the geographic localization (Delacuvellerie et al., 2019; Delacuvellerie et al., 2021; Oberbeckmann et al., 2021) are factors that strongly impact the microbial community.

\subsection{Polymer degradation}

Few reads of Alcanivorax were detected in the metagenomic data on three samples represented by both polymer chemical composition: ZI_1, ZI_4 and ZI_7 (Table S5). This genus is known for its capacity to degrade alkane and could be involved in PE degradation (Sabirova et al., 2011; Delacuvellerie et al., 2019). Therefore, enzymes involved in the degradation of PE or PP depolymerization products were investigated in all samples (Fig. 4). Indeed, PP and PE degradation is based on the alkane degradation pathway, which is characterized by key functions such as alkane hydroxylase (i.e., AlkB1 or AlkB2, involved in the oxidation of alkane chains of $\mathrm{C} 5$ to $\mathrm{C} 12$ and $\mathrm{C} 8$ to $\mathrm{C} 16$, respectively), the cytochrome P450, the ferredoxin and the ferredoxin reductase (Schneiker et al., 2006). Interestingly, three of these enzymes (i.e., ferredoxin, ferredoxin reductase and Acyl CoA-dehydrogenase) were detected in our metaproteomes and were expressed by Cyanobacteria, Bacteroidetes and unclassified organisms (Table S6). However, the central enzymes involved in the alkane degradation pathway (i.e., AlkB1 or AlkB2) were not detected. Moreover, the acyl CoA-dehydrogenase and the ferredoxin proteins are also involved in the $\beta$-oxidation and the photosystem processes, respectively. Therefore, we cannot confirm that the alkane degradation pathway was expressed in our samples. Previous studies have suggested that microbial organisms present in the plastisphere use plastics as a physical growing support and do not degrade them, as this requires a lot of energy (Krueger et al., 2015), and because of the large amount of carbon available in the environment (Delacuvellerie et al., 2019; Oberbeckmann et al., 2021).

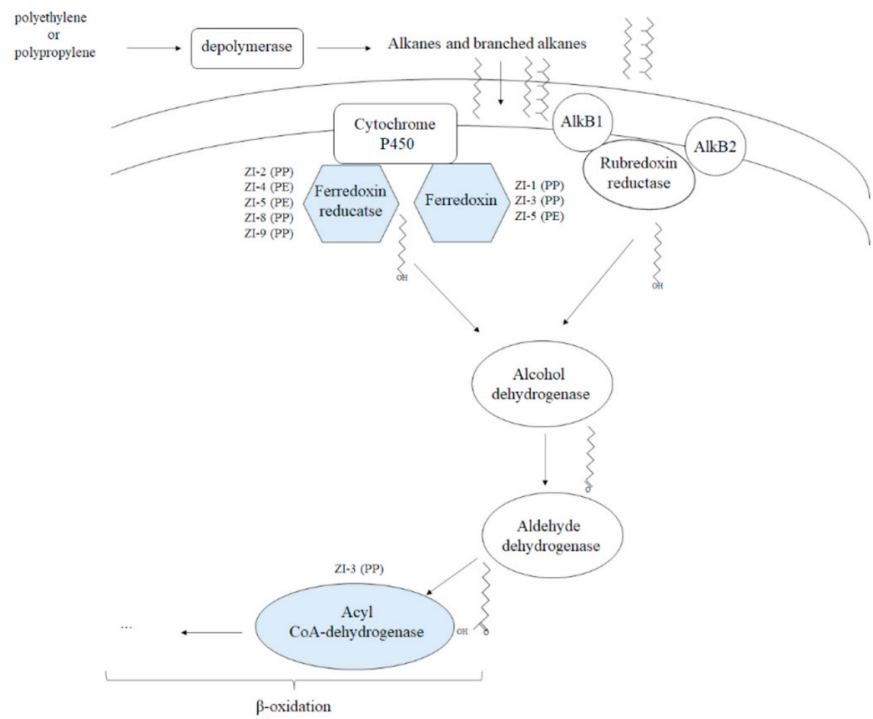

Fig. 4. Alkane degradation pathway. Our metagenomic data has been screened for enzymes involved in the pathways for alkane degradation. Enzymes found in our data are in blue, the name of the sample that contains these enzymes is written next the enzyme name. The illustration is based on the alkane pathway degradation from Sabirova et al. (2011) and Oberbeckmann et al. (2021). This schema does not contain the entire pathways but is focused on the potential enzymes involved in the initial degradation of polyethylene and polypropylene. (For interpretation of the references to colour in this figure legend, the reader is referred to the Web version of this article.)
The selection of degrader microorganisms of xenobiotic products or polymers in the plastisphere was studied. A previous study highlighted the presence of a gene involved in the xenobiotic degradation from metagenome on plastic, such as protocatechuate 3,4-dioxygenase (Bryant et al., 2016). Several of these proteins were present in our metagenomic analysis but in the metaproteome, only ethylbenzene dehydrogenase was found on the ZI-1 sample. Even though the microbial metagenomes contained genes involved in xenobiotic degradation, few bacteria expressed these proteins.

\subsection{Microbial pathogen in the plastisphere}

In our metagenomes, human pathogenic bacteria were mainly represented by the Vibrio (up to $88 \%$ of the total reads). Others pathogenic bacteria, such as Salmonella, accounted for only $2 \%$ of the total reads at genus level. The high abundance of Vibrionaceae could be explained by the fact that this sampling environment, i.e., pebble beach, is usually exposed to high concentrations of biological waste, which promote the development of these bacteria. To better understand the role of pathogenic species in the plastisphere, we studied the abundance of Vibrio as it was the most abundant pathogenic genus in both the metagenomes and the metaproteomes (Fig. 5). The most abundant species across all samples was Vibrio splendidus (reaching up to $70 \%$ of the total reads). Vibrio splendidus is a dominant planktonic species in temperate marine environments and is often involved in commensalism and pathogenic interactions with animals, such as oysters and clams (Gay et al., 2004; Le Roux and Austin, 2006). The proteins expressed by the Vibrionaceae family have been studied to highlight virulence-related processes (Fig. S9). In the present study, most abundant Vibrionaceae proteins were characterized as $50 \mathrm{~S}$ and $30 \mathrm{~S}$ ribosomal protein functions. In case of virulence, proteins involved in the type IV and VI secretory systems, the pilus assembly as well as chemotaxis protein are usually highly expressed. These proteins were not observed for the Vibrionaceae family, these results were in accordance with previous proteomic study (Oberbeckmann et al., 2021), where proteins involved in pathogenicity-related mechanisms were not found. All of our results seem to indicate that pathogenic organisms are very abundant on our plastics but are in dormancy with their harmful-related mechanisms not being active.

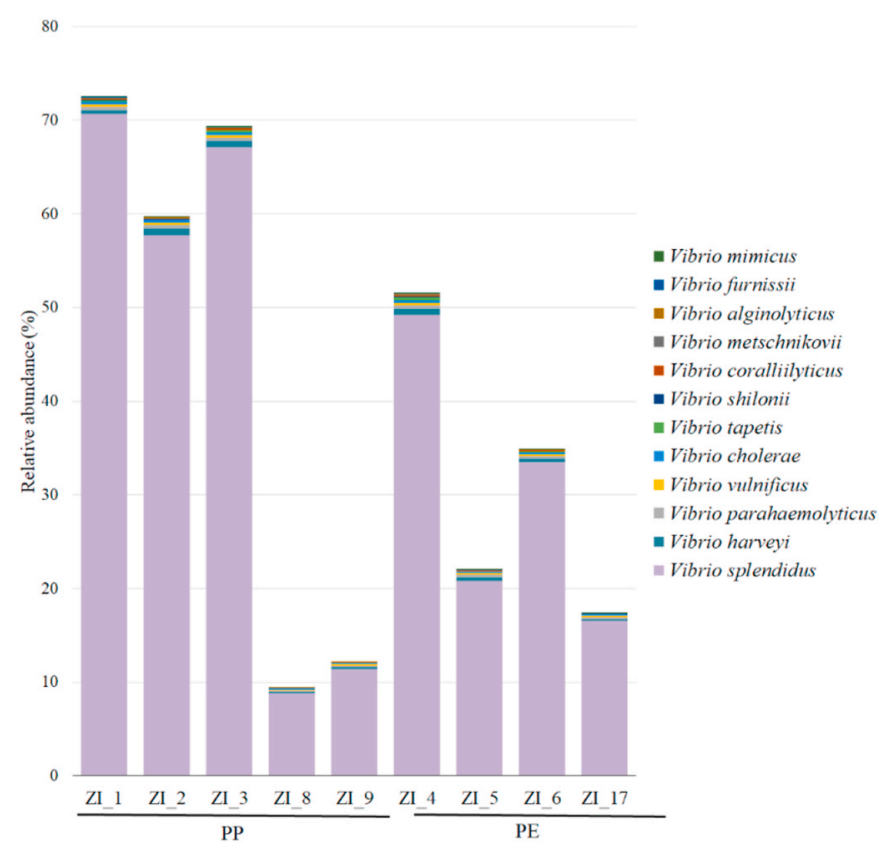

Fig. 5. Relative abundance calculated according to the number of reads from our metagenomic data of pathogenic species belonging of the Vibrio genus. 


\section{Conclusion}

This work provided valuable insights into the structure and the functioning of the plastisphere of PP and PE polymers. Metagenomic and metaproteomic analyzes confirmed that the plastispheres were organized as self-sufficient ecosystems. Interestingly, the polymer chemical composition was not found to significantly impact the plastisphere structure and functioning. Proteins of bacteria known to be involved in plastic degradation were not detected and bacterial pathogens seemed to be in a state of dormancy. These observations suggested that plastic debris surfaces are not undergoing degradation and are rather used as a new growth support and transport vessel for a broad range of taxa including pathogenic bacteria. Supplementary studies focusing the microorganisms from plastic debris evolving in different geographical locations and environmental conditions (floating plastics $v s$ on the sediment) should be carried out to further decipher the functioning of the plastispheres.

\section{Credit author statement}

Alice Delacuvellerie: Methodology, Formal analysis, Investigation, Writing - original draft preparation, Conceptualization. Augustin Géron: Writing - review \& editing, Methodology, Formal analysis. Sylvie Gobert: Writing - review \& editing, Resources; Ruddy Wattiez: Supervision, Resources, Writing - review \& editing, Conceptualization, Methodology.

\section{Declaration of competing interest}

The authors declare that they have no known competing financial interests or personal relationships that could have appeared to influence the work reported in this paper.

\section{Acknowledgments}

This study is funded by the Fund for Scientific Research (F.R.S-FNRS) FC 23347. We thank the Polymer and Composite Materials Department (UMONS) for the utilization of the ATR-FTIR. We thank Dr. Johannes Werner for assisting in the protein search data base creation. This study was sponsored by the "Belgian Fund for Scientific Research (Grand equipment-F.R.S-FNRS)". The bioprofiling platform used for the analyses was supported by the European Regional Development Fund and the Walloon Region, Belgium.

\section{Appendix A. Supplementary data}

Supplementary data to this article can be found online at https://doi. org/10.1016/j.envpol.2021.118678.

\section{References}

Amaral-Zettler, L.A., Zettler, E.R., Mincer, T.J., 2020. Ecology of the plastisphere. Nat. Rev. Microbiol. 18 (3), 139-151.

Amin, S.A., Parker, M.S., Armbrust, E.V., 2012. Interactions between diatoms and bacteria. Microbiol. Mol. Biol. Rev. 76, 667-684.

Barnes, D.K.A., 2002. Invasions by marine life on plastic debris. Nature 416 (April), 808-809.

Bolger, A.M., Lohse, M., Usadel, B., 2014. Trimmomatic: a flexible trimmer for Illumina sequence data. Bioinformatics 30, 2114-2120.

Bryant, J.A., Clemente, T.M., Viviani, D.A., Fong, A.A., 2016. Diversity and activity of communities inhabiting plastic debris in the north pacific gyre. mSystems 1 (3),

Buchfink, B., Xie, C., Huson, D.H., 2015. Fast and sensitive protein alignment using DIAMOND. Nat. Methods 12 (1), 59-60.

Colwell, R.R., Huq, A., 1994. In: Wachsmuth, I.K., Blake, P.A., Olsvik, O. (Eds.), Vibrios in the Environment: Viable but Non-culturable Vibrio cholerae. Vibrio cholerae and Cholera: Molecular to Global Perspectives. ASM Press, Washington, DC), pp. 117-133.

Cózar, A., Sanz-Martín, M., Martí, E., González-Gordillo, J.I., Ubeda, B., Gálvez, J.Á., et al., 2015. Plastic accumulation in the Mediterranean sea. PLoS One 10 (4).
Debroas, D., Mone, A., Halle, A., 2017. Plastics in the North Atlantic garbage patch: a boat-microbe for hitchhikers and plastic degraders. Sci. Total Environ. 599-600, 1222-1232.

Delacuvellerie, A., Cyriaque, V., Gobert, S., Benali, S., Wattiez, R., 2019. The plastisphere in marine ecosystem hosts potential specific microbial degraders including Alcanivorax borkumensis as a key player for the low-density polyethylene degradation. J. Hazard Mater. 380, 120899.

Delacuvellerie, A., Benali, S., Cyriaque, V., Moins, S., Raquez, J.-M., Gobert, S., Wattiez, R., 2021. Microbial biofilm composition and polymer degradation of compostable and non-compostable plastics immersed in the marine environment. J. Hazard Mater. 126526, 0304-3894.

De Tender, C.A., Devriese, L.I., Haegeman, A., Maes, S., Ruttink, T., Dawyndt, P., 2015. Bacterial community profiling of plastic litter in the Belgian part of the North Sea. Environ. Sci. Technol. 49, 9629-9638.

De Tender, C.A., Devriese, L.I., Haegeman, A., Maes, S., vangeyte, J., Cattrijsse, A., Dawyndt, P., Ruttink, T., 2017. The temporal dynamics of bacterial and fungal colonization on plastic debris in the North Sea. Environ. Sci. Technol. 49, 9629-9638.

Galgani, F., Leaute, J.P., Moguedet, P., Souplet, A., Verin, Y., Carpentier, A., Vilar, J. 2000. Litter on the sea floor along European coasts. Mar. Pollut. Bull. 40 (6), 516-527.

Garcés, E., Camp, J., 2003. Drifting plastic debris as a potential vector for dispersing Harmful Algal Drifting plastic debris as a potential vector for dispersing Harmful Algal Bloom ( HAB ) species. Sci. Mar. 67 (1), 107-111.

Gay, M., Berthe, F.C., Le Roux, F., 2004. Screening of Vibrio isolates to develop an experimental infection model in the Pacific oyster Crassostrea gigas. Dis. Aquat. Org. $59,49-56$

Géron, A., Werner, J., Wattiez, R., Lebaron, P., Matallana-Surget, S., 2019. Deciphering the functioning of microbial communities: shedding light on the critical steps in metaproteomics. Front. Microbiol. 10.

Geyer, R., Jambeck, J.R., Law, K.L., 2017. Production, use, and fate of all plastics ever made. Sci. Adv. 3, e1700782.

Glass, E.M., Wilkening, J., Wilke, A., Antonopoulos, D., Meyer, F., 2010. Using the Metagenomics RAST Server (MG-RAST) for Analyzing Shotgun Metagenomes. CSH protocols.

Hammer, Ø., Harper, D.A.T., Ryan, P.D., 2001. Paleontological statistics software package for education and data analysis. Palaeontol. Electron. 4 (1), 9-18, 7.

Huson, D.H., Beier, S., Flade, I., Górska, A., El-Hadidi, M., Mitra, S., et al., 2016. MEGAN community edition-interactive exploration and analysis of large-scale microbiome sequencing data. PLoS Comput. Biol. 12, e1004957.

Kirstein, I.V., Kirmizi, S., Wichels, A., Garin-fernandez, A., Erler, R., Martin, L., 2016. Dangerous hitchhikers ? Evidence for potentially pathogenic Vibrio spp . on microplastic particles. Mar. Environ. Res. 120, 1-8.

Kirstein, I.V., Wichels, A., Krohne, G., Gerdts, G., 2018. Mature biofilm communities on synthetic polymers in seawater - specific or general. Mar. Environ. Res. 142, 147-154, 2018.

Krueger, M.C., Harms, H., Schlosser, D., 2015. Prospects for microbiological solutions to environmental pollution with plastics. Appl. Microbiol. Biotechnol. 99 (21), 8857-8874.

Le Roux, F., Austin, B., 2006. Vibrio splendidus. In: Thompson, F.L., Austin, B., Swings, J. (Eds.), The Vibrios. American Society for Microbiology Press, Washington, DC, USA, pp. 285-296.

Leroy, B., De Meur, Q., Moulin, C., Wegria, G., Wattiez, R., 2015. New insight into the photoheterotrophic growth of the isocytrate lyase-lacking purple bacterium Rhodospirillum rubrum on acetate. Microbiology 161, 1061-1072.

Munari, C., Scoponi, M., Mistri, M., 2017. Plastic debris in the Mediterranean sea : types , occurrence and distribution along adriatic shorelines. Waste Manag. https://doi.org/ 10.1016/j.wasman.2017.05.020.

Oberbeckmann, S., Loeder, M.G.J., Gerdts, G., Osborn, A.M., 2014. Spatial and seasonal variation in diversity and structure of microbial biofilms on marine plastics in Northern European waters. Microb. Ecol. 90, 478-492.

Oberbeckmann, S., Osborn, A.M., Duhaime, M.B., Carpenter, E., Smith, K., Colton, J., Yamaji, H., 2016. Microbes on a bottle: substrate, season and geography influence community composition of microbes colonizing marine plastic debris. PLoS One 11 (8), e0159289. https://doi.org/10.1371/journal.pone.0159289.

Oberbeckmann, S., Kreikemeyer, B., Labrenz, M., 2018. Environmental factors support the formation of specific bacterial assemblages on microplastics. Front. Microbiol. 8 .

Oberbeckmann, S., Bartosik, D., Huang, S., Werner, J., Hirschfeld, C., Wibberg, D., Heiden, S.E., Bunk, B., et al., 2021. Genomic and Proteomic Profiles of Biofilms on Microplastics Are Decoupled from Artificial Surface Properties. Environmental microbiology.

Ogonowski, M., Motiei, A., Ininbergs, K., Hell, E., Gerdes, Z., Udekwu, K.I., et al., 2018. Evidence for selective bacterial community structuring on microplastics. Environ. Microbiol. 20, 2796-2808.

Pinnell, L.J., Turner, J.W., Turner, J.W., 2019. Shotgun Metagenomics Reveals the Benthic Microbial Community Response to Plastic and Bioplastic in a Coastal Marine Environment, vol. 10.

Pinto, M., Langer, T.M., Thorsten, H., Hofmann, T., Herndl, G.J., 2019. The composition of bacterial communities associated with plastic biofilms differs between different polymers and stages of biofilm succession. PLoS One.

Pruitt, K.D., Tatusova, T., Maglott, D.R., 2007. NCBI reference sequences ( RefSeq ): a curated non-redundant sequence database of genomes, transcripts and proteins. Nucleic Acids Res. 35, 61-65.

Ritchie, M.E., Phipson, B., Wu, D., Hu, Y., Law, C.W., Shi, W., Smyth, G.K., 2015. Limma powers differential expression analyses for RNA-sequencing and microarray studies. Nucleic Acids Res. 43, e47. 
Rho, M., Tang, H., Ye, Y., 2010. FragGeneScan: predicting genes in short and error-prone reads. Nucleic Acids Res. 38 e191-e191.

Sabirova, J.S., Becker, A., Lünsdorf, H., Nicaud, J.-M., Timmis, K.M., Golyshin, P.N., 2011. Transcriptional profiling of the marine oil-degrading bacterium Alcanivorax borkumensis during growth on n-alkanes. FEMS (Fed. Eur. Microbiol. Soc.) Microbiol. Lett. 319 (2), 160-168.

Schneiker, S., dos Santos, V., Bartels, D., et al., 2006. Genome sequence of the ubiquitous hydrocarbon-degrading marine bacterium Alcanivorax borkumensis. Nat. Biotechnol. 24, 997-1004.

Virsek, M.K., Lovsin, M.N., Koren, S., Krzan, A., Peterlin, M., 2017. Microplastics as a vector for the transport of the bacterial fi sh pathogen species Aeromonas salmonicida. Mar. Pollut. Bull. 125, 301-309.
Wang, Y., Naumann, U., Wright, S.T., Warton, D.I., 2012. Mvabund - an R package for model-based analysis of multivariate abundance data. Methods Ecol. Evol. 3, $471-474$.

Werner, J., Geron, A., Kerssemakers, J., Matallana-Surget, S., 2019. mPies: a novel metaproteomics tool for the creation of relevant protein databases and automatized protein annotation. Biorxiv.

Zettler, E.R., Mincer, T.J., Amaral-Zettler, L.A., 2013. Life in the "plastisphere": microbial communities on plastics marine debris. Environ. Sci. Technol. 47 (13), $7137-7146$. 